\title{
Improvement of broadband seismic station installations at the Observatoire de Grenoble (OSUG) seismic network
}

\author{
M. Langlais, B. Vial, and O. Coutant \\ ISTerre, CNRS, Universite Joseph Fourier, Grenoble, France \\ Correspondence to: M. Langlais (mickael.langlais@ujf-grenoble.fr) \\ Received: 31 October 2012 - Revised: N.A. - Accepted: 4 December 2012 - Published: 30 April 2013
}

\begin{abstract}
We describe in this paper different improvements that were brought to the installation of seismic broadband stations deployed by the Observatoire de Grenoble (OSUG) in the northern French Alps. This work was realized in the frame of a French-Italian ALCOTRA project (RISE), aimed at modernizing the broadband seismic networks across our common border. We had the opportunity with this project to improve some of our seismic recording sites, both in term of sensor installation quality, and in term of reliability. We detail in particular the thermal and barometric protection system that we designed and show its effect on the reduction of long period noise above $20 \mathrm{~s}$.
\end{abstract}

\section{Introduction}

ISTerre earth science laboratory at Observatoire de Grenoble (ISTerre-OSUG) has been running dense seismic networks in the French Alps for more than twenty years. From the Sismalp short period network (Thouvenot et al., 1990) to the regional broadband and accelerometric network RAP-ISTerre, (Péquegnat et al., 2008), the ISTerre laboratory has maintained a continuous development and evolution of its networks. These continuous efforts aim at improving the quality of the seismicity determination (localization and magnitude), and a better understanding of site effects in the context of alpine valleys (e.g. Guéguen et al., 2007; Causse et al., 2009). During the last three years (2009-2011), the ISTerre-OSUG laboratory has conducted a major upgrade of its short period and broadband seismic networks in collaboration with Géosciences Azur earth science laboratory and University of Genova. A regional INTERREG/ALCOTRA funded project, RISE (exchange network for seismic data and informations) was dedicated to the joint improvement and upgrade of alpine seismic network across the French-Italian border. The first aim was to build an uniform distribution of continuous recording broadband stations, at a high technical standard, on both sides of the Alps. The second objective was to improve data and information exchange (speed, quality) between the partners using again modern exchange protocols. For the ISTerre technical team in charge of geophysical networks, the project was a unique opportunity to work on the improvement of its seismic installations and to prepare the future RESIF (Pedersen, 2009) permanent network deployment. This paper describes how we improve (i) the quality of sensor installation and (ii) the monitoring of the new equipment in order to reduce maintenance time and field interventions. In a first part, we describe the different geophysical networks operated by ISTerre, then we detail the different technical improvements brought to our new sites.

\section{ISTerre-OSUG permanent networks}

ISTerre is in charge of 99 permanents Seismic/Geodetic sites in the northern western French Alps. These permanent sites belong to four different networks, managed by the same technical group. These networks are the regional contribution to four national networks: Strong motion French Accelerometric Network (RAP); French seismic network (RLBP/RéNaSS); French Geodedic Network (RENAG) and the new French landslide monitoring network (OMIV). In addition, ISTerre operates a short period seismic monitoring network (Sismalp) dedicated to the study of the local Alpine seismicity (e.g. Thouvenot et al., 2003). Figure 1 shows the geographical repartition of these 99 stations.

The permanent-network technical group at ISTerre is comprised of seven part-time engineers assisted by three researchers. A recent estimate of the time needed to maintain 


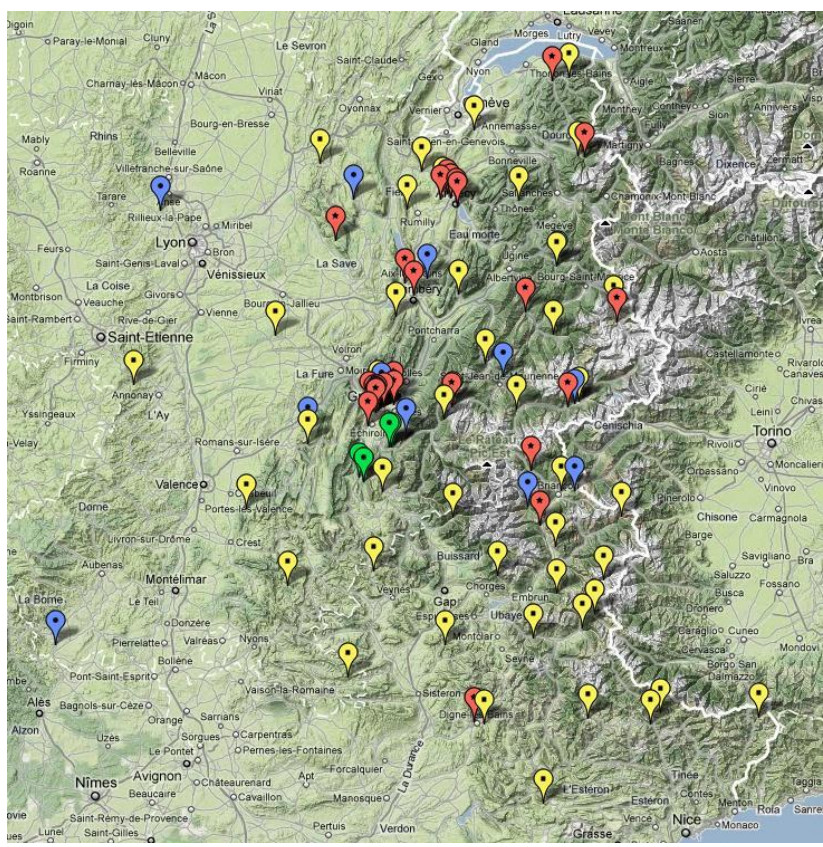

Fig. 1. Geographical distribution of the 99 permanent sites that are operated by ISTerre accross the French northern Alps. 33 StrongMotion (SM) stations from the French Accelerometric Network (RAP) - (red); 37 Short-Period (SP) + 7 Broad-Band (BB) stations from the RLBP/Sismalp network - (yellow); 16 GPS from the French Geodedic Network (RENAG) - (blue); 6 Mid-Band stations from the French landslides network (OMIV) - (green).

ISTerre sites shows that about 200 person-days are spent per year on field interventions. A key issue for our group is then to reduce this work. This was one of the technical objective of the RISE project: move selected short period, strong motion, and geodetic separated sites to common sites. This work was done after a careful examination of the networks and their specific scientific objectives. The strong-motion network sites for instance are chosen (i) to estimate site effects at location associated with soft superficial geological layers and hard rock reference sites, these locations were not considered in our project, or (ii), to complement velocimeter recordings that may saturate in case of close seismic event. We also took in account the position of the new stations deployed in Switzerland (SED network) and in Val D'Aoste (Italy).

Figure 2 shows an example of such saturation that was observed at SURF station. The magnitude 4.4 Barcelonnette earthquake (26 February 2012), located $10 \mathrm{~km}$ away from SURF, has been recorded few months after a short period Sismalp site was upgraded to a mixed broadband - Strong Motion sensor equipment with continuous recording.

The INTERREG/ALCOTRA RISE project finally allowed us to upgrade seven old sites and install a new one, while eliminating four isolated sites.

\section{Site upgrade overview}

One important aspect of this project was to improve the quality of the new sites in terms of reliability and in term of immunity to noise perturbations. To improve the robusteness, we choose to install digital acquisition systems (das) with high dynamic continuous acquisition, local storage on flash memory and a real-time transmission of the data. This real-time access allows to closely monitor the status of our sites and to automatically process different kind of informations: available stations, alarm triggering, automatic detection and localization of seismic events, data quality control,.... We use TCP/IP connectivity between the stations and our institute by mean of different technologies depending on what was available and robust for each site (DSL/3G/UMTS/RNIS). We also deployed on some site remote monitoring and control systems (such as AKCPSensorProbe $^{\mathrm{TM}}$ and Delta-Dore Typhone ${ }^{\mathrm{TM}}$ ) to enhanced the supervising of these sites (i.e. battery voltage, main power supply...) and to produce remote actions on the system (i.e.: modem or DAS hard reboot). These new facilities were installed in electrical enclosures compliant with the actual french standards (NF C 15-100) providing electrical and lightning protections. The Fig. 3 gives a synoptic overview of our new facilities.

It must be noted that the technological evolution from our oldest installation and the newest one is important as we changed from 12/16 bits triggered dialup recorders to 24 bits continuous recording/transmitting systems. We payed a particular attention to the sensors wiring (very flexible shielded cables with shielded twisted pairs for each channel), to limit noise perturbation due to EMC or cross-talk between channels. Moreover, the cables were attached to the ground to limit spread of vibrations to the sensors. We decided to take a particular care about broadband velocity sensor insulation considering their sensitivity to noise induced by thermal and barometric variations (see Zirn and Widmer (1995), Beauduin et al. (1996), Stutzmann et al. (2000)). We detail in the Sect. 4 the aluminium cover that was designed for this purpose. The new instruments transmit continuous real-time seismograms using the Seedlink protocol (Hanka et al., 2000) to gather and share data flows in realtime with other observatories (GéoSciencesAzur/OCA, IPGP/Goscope, DIPTERIS, SED/ETHZ...). These signals are used at ISTerre for data quality control and seismic event detection. We have implemented software solutions such as EarthWorm (Johnson et al., 1995) for automatic detection purpose, Seiscomp3 (see Weber et al. (2007) and Saul et al. (2010)) for real time monitoring (data quality-checking, automatic localisation, real time signal visualisation), Nagios (Barth, 2010) for network connectivity control, PQLx (McNamara and Boaz, 2006) for automatic data quality processing, and also several scripts developed to control the state of health of our installations (using Python, Matlab, bash...). All these softwares helps us to have a near real time status 


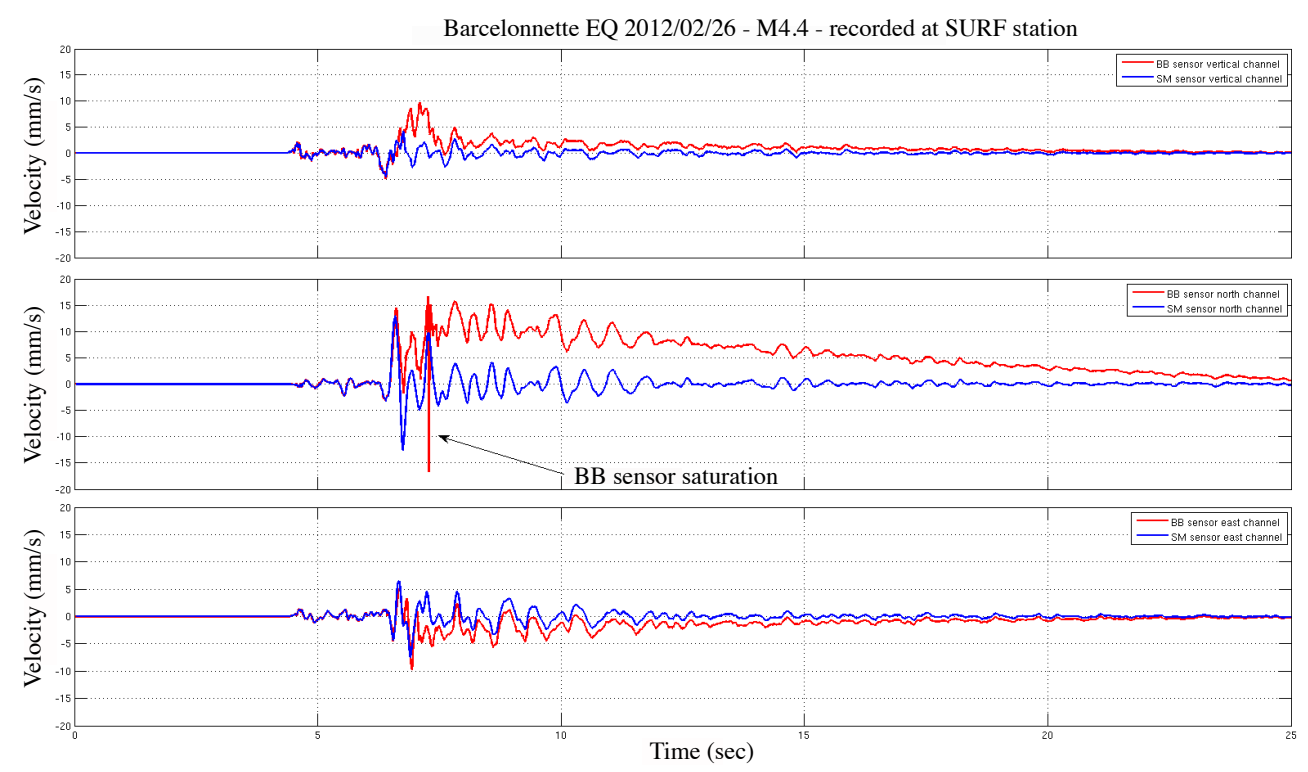

Fig. 2. Accelerometer (blue) and broadband seismometer (red) recordings of the Barcelonette Mag 4.4 earthquake, located $10 \mathrm{~km}$ away SURF station. All traces converted to velocity.

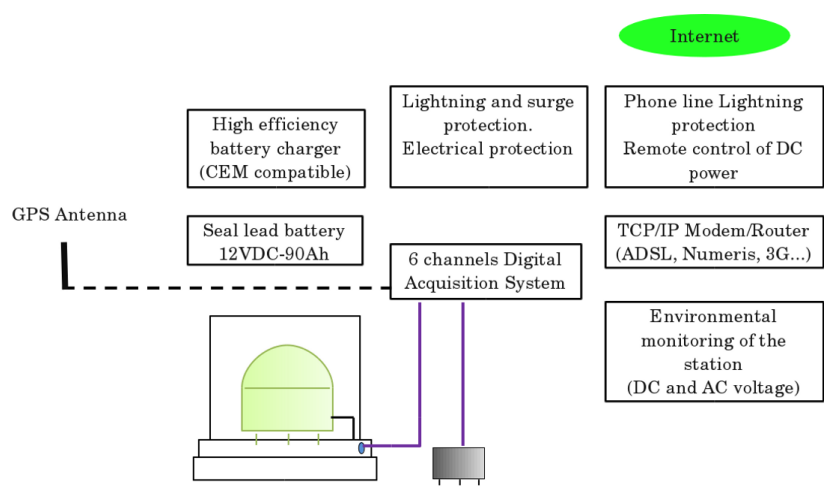

Fig. 3. schematic of our new installations.

of the geophysical networks. Alerts in case of issue are sent to the technical team (emailing). We already experienced operational improvement thanks to this monitoring system that helps us to detect quickly any issue on our remote sites and to solve them before any lose of data.

\section{TANK protection: aluminium alloy bolted on granite plate}

\subsection{Design}

We decided to pay a particular attention to the installation of our broadband sensors based on the community experience and guidelines for broadband sensors installations improvements (see Trnkoczy et al. (2012), Forbriger (2012) and Leroy et al. (2010)). We then developed a dedicated protec- tion system called TANK. This system has been designed from the CASSIS solution developed for GEOSCOPE network at IPGP by Sebastien Bonaimé and Christophe Brunet (personal communication, 2009). The CASSIS system itself is inspired from the so-called Lennartz STS-2 shielding kit and the Stuttgart Shielding (Widmer-Schnidrig and Kurrle, 2006) and presents three advantages in our case: multiple sensor compatibility (Streckeisen STS-2, Nanometrics Trillium-120, and Trillium-240), cable gland (no extra connector), orientation marks on the granite plate.

We decided to design a new cover to improve the mounting of the system and the humidity/barometric insulation. The Fig. 4 (top) shows a diagram of the TANK protection.

\subsection{Measurement of pressure and thermal insulation}

We have tested the pressure insulation efficiency of the TANK using three different methods: (i) numerical simulation of the deformation due to a pressure load, (ii) pressure response to a step variation, (iii) natural pressure variation response.

For the first method we use the software SolidWorks to study the mechanical response of the TANK to an air pressure load. During the numerical simulation (finite element method) (Fig. 4, bottom) we simulate a $+25 \mathrm{hPa}$ variation of the external pressure, assuming constant internal temperature. We calculate the maximum deformation of the cover bolted due to this over-pressure and observe a maximum deformation of $0.316 \mathrm{~mm}$ on the top plate of the cylinder. This deformation would induce an internal over-pressure of $0.466 \mathrm{hPa}$ and thus reduces the external over-pressure effect 


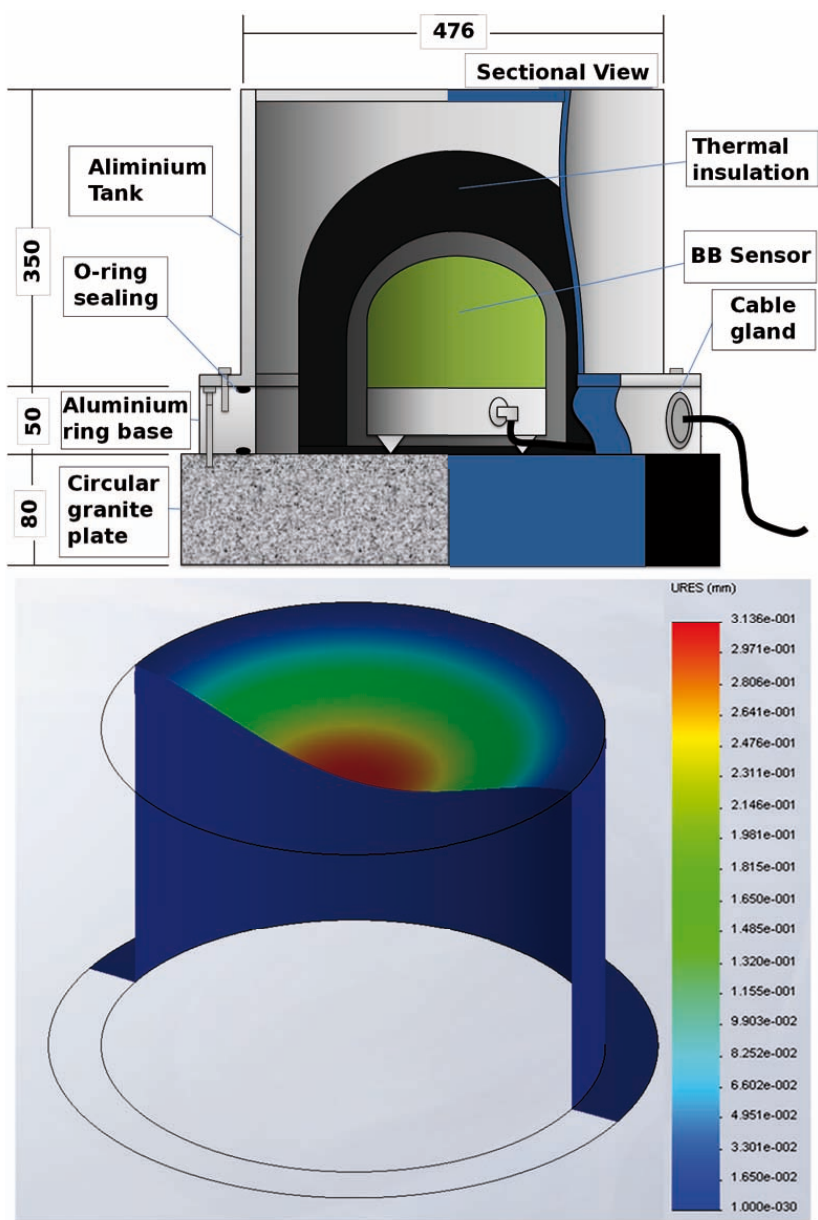

Fig. 4. Diagrams showing the details of the Tank protection (top) and the cover deformation induced by a barometric pressure increase outside the TANK (bottom).

by a factor of 55 . This is of course true in the case of a perfect sealed installation, without any thermal variation.

For the second evaluation method, the tank is submitted to a rapid, step-like, barometric pressure decrease (from the mountains down-to Grenoble City: $-1500 \mathrm{~m}$ in elevation). Figure 5 displays the pressure leakage between internal and external pressures. From this test, the pressure leakage time constant can be estimated to $4 \mathrm{~h}$. The system is not airtight but is able to filter hourly pressure variations.

For the third method we leave the tank installed during 4 days in a stable thermal room, observing the inside and outside pressure variation. These pressure curves are presented on the Fig. 6. The outside pressure variations are low-pass filtered by the TANK. While the long period variations are not filtered because air tightness of the TANK is not achieved, faster pressure variations (few hours) are well filtered by the TANK. One way to improve the sealing of the system will be to improve the mechanic of the cable through the gland.

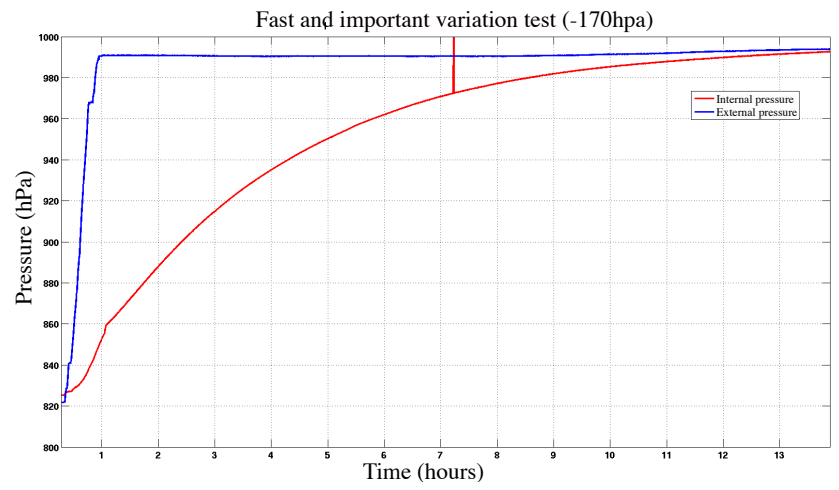

Fig. 5. Effect of a rapid pressure change applied outside the TANK protection.

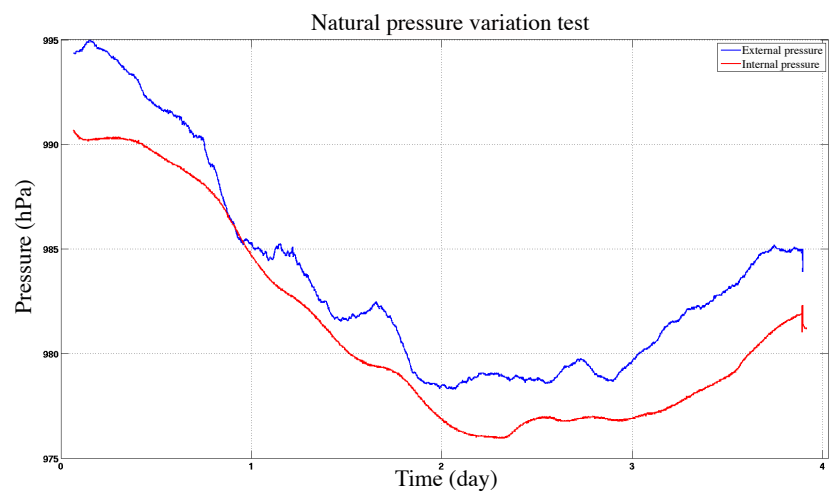

Fig. 6. Effect of a slow pressure change applied outside the TANK protection.

\subsection{Noise comparison}

A different way to evaluate the efficiency and the benefit of the TANK is to perform noise comparisons on the sites where the shielding has been deployed. We evaluate the benefit of the TANK by comparing the data recorded before and after the installation of this barometric/thermal insulation. This comparison has been achieved for five different stations, over at least one year of data recordings. The period of observation without and with the insulation are different for each site. We present in Fig. 7 one example for site SURF that is demonstrative of all observations. The three steps of the analysis are (i) conversion from mseed+dataless in SAC format (RDSEED software provide by IRIS), (ii) deconvolution of the instrumental response to acceleration and decimation (SAC Goldstein et al. (2003)), (iii) Daily Power Spectral Density estimation (Welch estimation Welch (1967)) and then plotting in a T/F style. The Fig. 7 shows the efficiency of the TANK on the noise reduction for periods greater than $20 \mathrm{~s}$. The improvements are mainly visible on vertical channels, because this solution could not obviously reject the noise due to local soil tilt on horizontal channels (deformation due to pressure/thermal variations). 


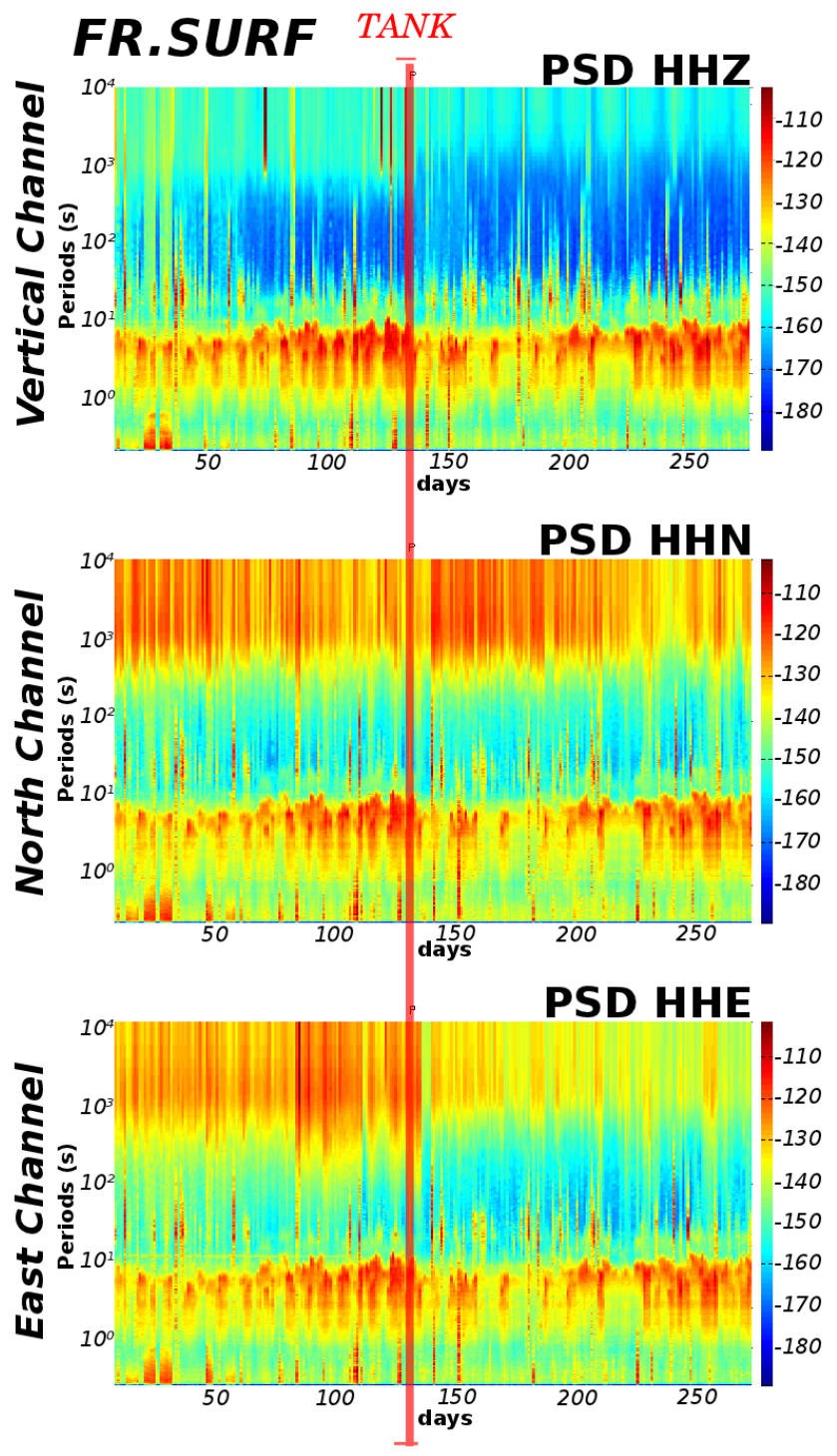

Fig. 7. Frequency/time power spectral density for the three Z, N and E components at SURF stations before and after the installation of the TANK thermal and barometric protection. The red vertical line corresponds to the date of the protection installation.

For periods above $20 \mathrm{~s}$, we have shown that the noise reduction is efficient over each channels from 5 to $20 \mathrm{~dB}$ for the stations analyzed operating different broadband sensors (Streckeisen STS-2, Nanometrics Trillium-120, and Trillium-240). We consider that the improvement are mainly due to (i) thermal insulation: smaller air volume and so less temperature fluctuation around the sensor, (ii) barometric insulation: the tanks acts as an low-pass filter to the pressure variations (few hours), (iii) general improvement of the sensor installation (granite plate, cable installation...). Those observations are consistent with the informations found in Forbriger (2012), and also personal communications from
J. M. Saurel(2012) and S. Bonaimé (2009) on the evaluation of the CASSIS shielding.

\subsection{Future designs/improvements}

We plan to go forward by designing dedicated internal and external thermal insulation (machined polyurethane foam), to improve the rigidity of the cover bolt, and to upgrade the gland for the cable with an air-tight system through the ring. We are also working on a simplest design to add protection on the SM sensors (Type Kinemetrics-Episensor) and BB/MB Velocimeter (Type Nanometrics-TrilliumCompact). This protection will allow a reduction of the influence of the thermal and barometric variations and will protect the sensors from the humidity. Recent personal communication from J. M. Saurel (2012) has shown long period noise reduction for the TrilliumCompact protected from thermal and barometric fluctuations (CASSIS shielding).

\section{Conclusions}

We have shown that, thanks to the improvement and modernization applied to some of our geophysical sites (installation up to date, continuous monitoring, real time transmission), we have clearly increased both the quality of the signals recorded and the efficiency of our seismic network. The particular care given to the installation of the broad band sensors and their wiring to the digital acquisition System (DAS) has been successful. We improve the noise recorded above $20 \mathrm{~s}$ from 5 to $20 \mathrm{~dB}$. This gain is mainly due to the installation of the dedicated insulation system named the TANK. This TANK system has several advantages (i) compatibility with at least three BB sensors types, (ii) mechanical assembly using an aluminum alloy ring (easy to install, no extra connector for the sensor, no putting to seal the cable), (iii) the aluminum cover bold design (assembly improving, nonmagnetic, cost reduction in series), (iv) the granite plate design (easy orientation for multiple BB sensors, stable and clean surface). This insulation improves the seismic signal quality because it reduces the thermal and barometric variations which are sources of noise perturbation for the broadband velocimeters. Further improvements of the sensor installation will include (i) addition of extra thermal insulation around the TANK and (ii) design of a new mechanic gear around the cable gland to enhanced the air-tightness of the system.

Acknowledgements. We gratefully acknowledge the members of ISTerre-OSUG who activally participated to the RISE project: N. Cotte, I. Douste-Bacqué, L. Jenatton, L. Piard, R. Bethoux. We also thank S. Bonaimé and J. M. Saurel for fruitfull technical discussion about broadband sensors insulation methods.

This work benefited from the financial support of the CNRS/INSU (Centre National de la Recherche/Institut des Sciences de l'Univers), the DIREN (Direction Régionale de l'ENvironnement) 
Rhône-Alpes, the Isère, Ain and Haute-Savoie General Councils and the FEDER (Fonds Européens de Développement Régional) in the framework of the RISE project in the ALCOTRA (Alpes Latines COopération TRAnsfrontalière) program (2007-2013).

This work has also been supported by a grant from LabEx OSUG@2020 (Investissements d'avenir ANR10 LABX56).

\section{References}

Barth, W.: Nagios: System-und Netzwerk-Monitoring, No Starch Press, 2010.

Beauduin, R., Lognonne, P., Montagner, J., Cacho, S., Karczewski, J., and Morand, M.: The effects of the atmospheric pressure changes on seismic signals or how to improve the quality of a station, B. Seismol. Soc. Am., 86, 1760-1769, 1996.

Causse, M., Chaljub, E., Cotton, F., Cornou, C., and Bard, P.: New approach for coupling k- 2 and empirical Green's functions: application to the blind prediction of broad-band ground motion in the Grenoble basin, Geophys. J. Int., 179, 1627-1644, 2009.

Forbriger, T.: New manual of seismological observatory practice, vol. CHAPTER 5: Recommendations for seismometer deployment and shielding, IASPEI, GFZ German Research Center for Geosciences, Potzdam, doi:10.2312/GFZ.NMSOP-2, 2012.

Goldstein, P., Dodge, D., Firpo, M., and Minner, L.: 85.5 SAC2000: Signal processing and analysis tools for seismologists and engineers, Int. Geophys., 81, 1613-1614, 2003.

Guéguen, P., Cornou, C., Garambois, S., and Banton, J.: On the Limitation of the H/V Spectral Ratio Using Seismic Noise as an Exploration Tool: Application to the Grenoble Valley (France), a Small Apex Ratio Basin, Pure Appl. Geophys., 164, 115-134, doi:10.1007/s00024-006-0151-x, 2007.

Hanka, W., Heinloo, A., and Jaeckel, K.: Networked seismographs: GEOFON real-time data distribution, 2000.

Johnson, C., Bittenbinder, A., Bogaert, B., Dietz, L., and Kohler, W.: Earthworm: A flexible approach to seismic network processing, Iris newsletter, 14, 1-4, 1995.

Leroy, N., Pardo, C., Bonaime, S., Stutzmann, E., and Maggi, A.: GEOSCOPE Observatory Recent Developments, in: AGU Fall Meeting Abstracts, Vol. 1, p. 2027, 2010.
McNamara, D. and Boaz, R.: PQLX: A software tool to evaluate seismic station performance, in: AGU Fall Meeting Abstracts, Vol. 1, p. 0236, 2006.

Pedersen, H.: RESIF: a French Integrated Antenna for the Observation of Earth Deformation, in: AGU Fall Meeting Abstracts, Vol. 1, p. 03, 2009.

Péquegnat, C., Guéguen, P., Hatzfeld, D., and Langlais, M.: The French accelerometric network (RAP) and national data centre (RAP-NDC), Seismol. Res. Lett., 79, 79-89, 2008.

Saul, J., Becker, J., Hanka, W., Heinloo, A., and Weber, B.: SeisComP 3-Where are we now?, in: EGU General Assembly Conference Abstracts, Vol. 12, p. 11074, 2010.

Stutzmann, E., Roult, G., and Astiz, L.: GEOSCOPE station noise levels, B. Seismol. Soc. Am., 90, 690-701, 2000.

Thouvenot, F., Fréchet, J., Guyoton, F., Guiguet, R., and Jenatton, L.: Sismalp: an automatic phone-interrogated seismic network for the western Alps, Cahiers du Centre Européen de Géodynamique et de Séismologie, 1, 10, 1990.

Thouvenot, F., Fréchet, J., Jenatton, L., and Gamond, J.: The Belledonne Border Fault: Identification of an active seismic strike-slip fault in the western Alps, Geophys. J. Int., 155, 174-192, 2003.

Trnkoczy, E., Bormann, P., Hanka, W., Holcomb, L., Nigbor, R., Shinohara, M., Suyehiro, K., and Shiobara, H.: New manual of seismological observatory practice, vol. CHAPTER 7: Site Selection, Preparation and Installation of Seismic Stations, IASPEI, GFZ German Research Center for Geosciences, Potzdam, doi:10.2312/GFZ.NMSOP-2, 2012.

Weber, B., Becker, J., Hanka, W., Heinloo, A., Hoffman, M., Kraft, T., Pahlke, D., Reinhardt, J., Saul, J., and Thoms, H.: SeisComautomatic and interactive real-time data processing, in: Geophysical Research Abstracts, Vol. 9, p. 09219, 2007.

Welch, P.: The use of fast Fourier transform for the estimation of power spectra: a method based on time averaging over short, modified periodograms, IEEE T. Acoust. Speech., 15, 70-73, 1967.

Widmer-Schnidrig, R. and Kurrle, D.: Evaluation of installation methods for Streckeisen STS-2 seismometers, 2006

Zïn, W. and Widmer, R.: On noise reduction in vertical seismic records below $2 \mathrm{mHz}$ using local barometric pressure, Geophys. Res. Lett, 22, 3537-3540, 1995. 